\title{
Paralelismo, convergencia y homología profunda en la biología: una propuesta conceptual*
}

\author{
Parallelism, Convergence, and Deep Homology in Biology: A Conceptual Proposal
}

\author{
Claudia Lorena García
}

\begin{abstract}
Resumen
En este trabajo analizo las siguientes nociones de homoplasia: paralelismo y convergencia, y realizo al mismo tiempo un examen ceñido de la noción de homología profunda, tal y como se usan en la morfología comparada, ya que existen muchas de caracterizar cada una de estas nociones que, además, muestran la relación que existe entre estas nociones y ciertas teorías generales acerca de las conexiones que presuntamente existen entre los factores que constriñen los cambios ontogenéticos y ciertas tendencias macroevolutivas que parcialmente determinan las rutas que toma la filogenia; en particular, me referiré a: (1) la idea de que el desarrollo es "como un embudo" (the funnel model); y (2) al modelo del desarrollo conocido como "el reloj de arena" (the hourglass model).
\end{abstract}

Palabras clave: desarrollo y filogenia - tendencias macroevolutivas - el modelo del embudo - el modelo del reloj de arena - homoplasia

\begin{abstract}
I examine two notions of homoplasy, to wit, parallelism and convergence, and pursue my analysis addressing another important phylogenetic notion, that of deep homology. My interest is to contribute to clarify the controversies concerning how these notions are used in comparative morphology, and also whether such notions should continue to be used at all. I will also show that there are some interesting and empirically grounded ways to characterize each of these notions. These characterizations show that there are strong connections between some of the causal factors that direct ontogenetic change and certain macroevolutionary tendencies; in particular, I will mention two of them: (1) the idea that development is like a funnel, and (2) the model of development known as the 'hourglass model'.

Keywords: development and phylogeny - macroevolutionary tendencies - the Funnel Model - the Hourglass Model homoplasy
\end{abstract}

\footnotetext{
* Recibido: 3 de Junio de 2016. Aceptado con revisiones: 5 de Diciembre de 2016.

${ }^{\dagger}$ Instituto de Investigaciones Filosóficas-Universidad Nacional Autónoma de México. Para contactar al autor, por favor, escribir a: clga1305@gmail.com.

Metatheoria 8(1)(2017): 57-69. ISSN 1853-2322.

(C) Editorial de la Universidad Nacional de Tres de Febrero. Publicado en la República Argentina.
} 


\section{Introducción}

En la biología comparada, caracteres semejantes presentes en dos o más taxones se consideran homólogos cuando derivan con ciertas modificaciones de un carácter que se encuentra en el ancestro más reciente común a todos ellos. En contraste cuando caracteres semejantes no cumplen con esta condición se consideran homoplásicos. Por ejemplo, las extremidades anteriores de los humanos y del resto de los mamíferos son homólogas ya que todas ellas son semejantes, y el ancestro común más reciente de todos los mamíferos tenía extremidades anteriores de las cuales nuestras extremidades anteriores derivaron con modificaciones. Por otra parte, las alas de los pájaros y las de los murciélagos (qua alas) son homoplásicas puesto que el ancestro común más reciente de estos grupos de organismos no tenía alas. ${ }^{1}$

Ahora bien, descubrimientos relativamente recientes en la biología del desarrollo muestran que la noción de homoplasia abarca una variedad de conexiones biológicas interesantes entre los rasgos de organismos pertenecientes a diferentes grupos, linajes, o especies. Usualmente, se hacen las siguientes distinciones entre los caracteres homoplásicos:

(a) Están aquellos caracteres que son paralelos; a grosso modo, son rasgos semejantes que no son homólogos pero que, en algún sentido, evolucionaron de una manera no independiente, ya que su semejanza se debe, por lo menos parcialmente, a una semejanza y/u homología de algunos factores que son, en algún sentido, "internos" a esos organismos; por ejemplo, factores ontogenéticos (por ejemplo, Simpson 1961, Hall 2003).

(b) Por otra parte, están aquellos caracteres que son convergentes cuyas semejanzas se explican principalmente como resultado de presiones ambientales semejantes.

Esta manera relativamente pre teórica de caracterizar la distinción entre paralelismo y convergencia no es completamente neutral desde un punto de vista conceptual puesto que muchos biólogos evolutivos asocian esta distinción simplemente con cuestiones acerca de la cercanía o lejanía filogenética de los taxones, especies o linajes que se están comparando, de manera tal que dos rasgos serían paralelos si no son homólogos aunque estructuralmente muy semejantes y los grupos que presentan esos rasgos son filogenéticamente cercanos; mientras que los rasgos serían convergentes solo si los grupos relevantes son lejanos. ${ }^{2}$ Más adelante mostraremos que, de aceptar la caracterización aquí propuesta de estos términos, la relación que existe entre paralelismo y cercanía podría bien ser probabilística y tener un trasfondo teórico más robusto.

Existe otra noción ampliamente usada en la biología que se aplica a aquellos caracteres de dos o más taxones que pueden o no ser semejantes pero que comparten algunas porciones de su desarrollo temprano. En estos casos se habla de la presencia de una homología profunda. En los últimos cuarenta años aproximadamente se han descubierto muchos rasgos que son profundamente homólogos pues sus

\footnotetext{
${ }^{1}$ En la literatura contemporánea de distintas ramas de la biología, existen diferentes nociones de homología; principalmente: (1) la noción táxica de homología, en la que se identifica la homología con sinapomorfia (de Pinna 1991), es decir, con la existencia de ciertos tipos de relaciones filogenéticas entre rasgos, relaciones que se evidencian por la existencia de un grupo monofilético, (2) la llamada 'homología biológica' según la cual (a grandes rasgos) la homología está constituida por relaciones de semejanzas sustanciales entre porciones de la ontogenia de los rasgos relevantes (Wagner 1989), y (3) la homología organizacional, que entiende a la homología como la existencia de semejanzas (a diferentes niveles) en la organización morfológica de los rasgos en cuestión y de otros rasgos que estén conectados con aquéllos (Muller 2003). De todas estas nociones de homología, la que tiende a ser más usada es la táxica, y básicamente es ésta con la que trabajaremos en este contexto. Una discusión más completa sobre a noción de homología se encuentra en García (2010).

${ }^{2}$ Véase Arendt y Reznick (2007), quienes hacen un recuento relativamente exhaustivo de las maneras en que paralelismo y convergencia se usan en la literatura en morfología animal comparada.
} 
desarrollos tempranos comparten genes y/o redes genéticas regulatorias, aun cuando los rasgos mismos no son homólogos. Más adelante presentaremos algunos ejemplos de cada uno de estos fenómenos.

Aquí examinaré estas dos nociones de homoplasia, al igual que la de homología profunda, tal y como se usan en la morfología comparada, ya que existen muchas controversias respecto a cómo caracterizarlas e, incluso, si vale la pena rescatarlas. También mostraré que existe una manera interesante y empíricamente asequible de caracterizar cada una de estas nociones que mostrando la relación que existe entre éstas y ciertas teorías generales acerca de las conexiones que presuntamente existen entre los factores que constriñen los cambios ontogenéticos y ciertas tendencias macroevolutivas que parcialmente determinan las rutas que toma la filogenia; en particular, me referiré a: (1) la idea de que el desarrollo es "como un embudo"; idea que está asociada a la noción de atrincheramiento generativo de William Wimsatt (1986), y (2) al modelo del desarrollo conocido como "el reloj de arena" (Sander 1983, Duboule 1994, Raff 1996).

\section{Nociones de paralelismo, convergencia y homología profunda morfológicos}

Como ya mencionamos, dentro de la categoría de homoplasia, algunos biólogos distinguen otras categorías, principalmente, el paralelismo y la convergencia; la homología profunda parece ser un caso un tanto distinto ya que se aplica a rasgos que pueden o no ser semejantes pero cuyos desarrollos tempranos comparten algunos factores cruciales.

Muchas de las caracterizaciones de la noción de paralelismo proponen que dos rasgos son paralelos si y solo si son semejantes y homoplásicos a un nivel de la jerarquía biológica (v.gr., en el nivel de la morfología), pero sus genes y/o sus redes genéticas regulatorias son homólogos (v.gr., Simpson 1961, Hall 2003, Scotland 2011). ${ }^{3}$ En contraste, se consideran convergentes aquellos rasgos homoplásicos y semejantes cuyos desarrollos no son homólogos ni en sus genes ni en sus redes genéticas. La idea central de la convergencia es que se trata de rasgos cuyas semejanzas se explican principalmente en términos de la presencia de presiones ambientales semejantes durante la fijación de tales rasgos en los linajes relevantes.

Sin embargo, uno de los problemas más serios que aquejan actualmente a esta manera de hacer la distinción entre paralelismo, convergencia y homología profunda es el siguiente: descubrimientos relativamente recientes en el campo de la biología molecular del desarrollo han mostrado que la mayoría de los organismos multicelulares comparten, en mayor o menor medida, porciones de sus genomas, y que algunas de las redes genéticas regulatorias de linajes tanto cercana como lejanamente relacionados están conservadas (Arendt \& Reznick 2007).

Por ejemplo, los desarrollos tempranos de los ojos de los artrópodos, de los moluscos e incluso de los vertebrados involucran el gen de control maestro Pax-6/eyeless; sin embargo, es ampliamente aceptado que los ojos de los artrópodos, de los moluscos y de los vertebrados no son homólogos. Y como éste hay muchísimos otros casos; los desarrollos de muchos rasgos de linajes lejanamente relacionados involucran genes homólogos y/o redes genéticas regulatorias homólogas.

\footnotetext{
${ }^{3}$ Hay un cierto consenso entre biólogos y filósofos de la biología que existen varios niveles de organización biologica, niveles que están causalmente interrelacionados, ordenados jerárquicamente, y que no son ni descriptiva ni explicativamente reducibles unos a otros. Adicionalmente, la mayoría también está de acuerdo que "la homología es un fenómeno que puede expresarse de manera independiente en muchos niveles distintos de la organización biológica." (Abouheif 1999, p. 215); en particular, que la homología (o la homoplasia) a un determinado nivel (digamos, el genético o el embriológico) no implica necesariamente homología (u homoplasia) a otros niveles (por ejemplo, el morfológico) (Laubichler 2000, Striedter \& Northcutt 1991). Hay muchos ejemplos de rasgos morfológicos que son homologos pero cuyos genes y redes genéticas regulatorias no lo son (Striedter \& Northcutt 1991, Hall 2003, Müller 2003, Sommer 2008).
} 
Pero entonces parecería que el fenómeno de convergencia -si se le entiende como el fenómeno de una evolución verdaderamente independiente que se explica principalmente en términos de presiones ambientales semejantes- es un suceso muy raro, por lo menos en la evolución de los metazoarios. Adicionalmente, si dijésemos -como lo hacen muchos biólogos (p.e., Shubin, Cliff \& Carroll 2009, Wake, Wake \& Specht 2011)- que el paralelismo ocurre en los casos de rasgos semejantes cuyos desarrollos involucran genes homólogos y/o redes genéticas regulatorias homólogas, entonces resultaría que casi todos (si no es que todos) los rasgos de la mayoría de los metazoarios serían casos de evolución paralela, y no habría lugar para la categoría de homología profunda. ¿Cómo entonces se podrían distinguir el paralelismo y la homología profunda, si ésta también se refiere a aquellos rasgos cuyos desarrollos involucran genes y/o redes genéticas homólogos? ¿Cómo caracterizar la convergencia de manera tal que ésta no sea un fenómeno extremadamente raro?

Recientemente, un filósofo de la biología, Russell Powell (2012), le ha dado una respuesta interesante a la pregunta sobre la naturaleza del paralelismo apelando a lo que, según él, es (presuntamente) una distinción entre causas especificas y causas generales propuesta por Kenneth Waters (2007). ${ }^{4}$ Usando esta distinción, Powell propone que entendamos al paralelismo de la siguiente manera: "Una homoplasia es paralelismo syss alguna [porción] tardía de su maquinaria del desarrollo es al mismo tiempo homóloga y causalmente específica” (Powell 2012, p. 15, mi énfasis).

Por razones de brevedad en esta presentación no explicaré por qué encuentro la propuesta específica de Powell inaceptable. Sólo mencionaré que el problema se encuentra en el hecho que las nociones de causalidad de Waters que Powell usa no pueden entenderse como nociones de causas específicas (en oposición a causas generales). ${ }^{5}$ Sin embargo, pienso que la propuesta general de Powell va en la dirección correcta.

\section{Reformulando el paralelismo, la convergencia y la homología profunda}

En la presente sección propondré una noción de especificidad causal más afín a mi propósito de formular una noción de paralelismo que sea adecuada. Para ello, presentaré primero una noción de causa, de James Woodward (2003), que después usaré para caracterizar una noción de causa específica.

\subsection{Causas}

Para caracterizar de manera clara la idea de que una causa es, en un contexto normal, específica para un cierto efecto, usaré la teoría manipulacionista de la causalidad de James Woodward, de acuerdo con la cual (a grandes rasgos) X causa Y si y solo si el valor de Y cambiaría al manipular el valor de X donde $\mathrm{X}$ y son variables que toman dos o más valores mutuamente excluyentes- ${ }^{6}$

\footnotetext{
${ }^{4}$ Digo 'presuntamente' puesto que la distinción que propone Waters (2007) no es, bajo ninguna interpretación, una distinción entre causas de hechos (o propiedades) específicos vs. causas de hechos (o propiedades) generales, que es lo que Powelll supone -algo que muestro en mi artículo "El paralelismo y la especificidad causal en el desarrollo tardío: examen de la propuesta de Russell Powell con respecto al paralelismo evolutivo" (en prensa)-.

${ }^{5}$ En mi ensayo "El paralelismo y la especificidad causal en el desarrollo tardío: examen de la propuesta de Russell Powell con respecto al paralelismo evolutivo" (en prensa) argumento estos puntos en detalle.

${ }^{6}$ Debemos notar que, para Woodward, las relaciones causales entre dos variables no necesitan ser invariantes sobre los valores de otras variables relevantes. Así, si estamos estudiando un fenómeno que involucra muchas variables causales $\left(\mathrm{C}_{1}, \ldots, \mathrm{C}_{\mathrm{n}}\right)$ en relación con un efecto E, bien podría ser el caso que los siguientes escenarios causales sean diferentes con respecto al efecto E: Tenemos una población $\mathrm{P}$ de organismos de cierta especie; en esta población, $\mathrm{C}_{1}$ toma el valor $\mathrm{c}_{1}{ }^{*}, \mathrm{C}_{2}$ toma el valor $\mathrm{c}_{2}{ }^{*}, \ldots$ y $\mathrm{C}_{\mathrm{n}}$ toma el valor $\mathrm{c}_{\mathrm{n}}{ }^{*}$. En $\mathrm{P}$ en este momento $\mathrm{E}$ toma el valor $\mathrm{e}^{*}$. Esto no quiere decir ni que necesariamente $\mathrm{C}_{1}$ sea causalmente específica de $\mathrm{E}$ (como veremos más adelante), ni que el valor $\mathrm{c}_{1}{ }^{*}$ siempre nos arroje $\mathrm{e}^{*}$ como resultado, puesto que un resultado diferente en $\mathrm{E}$ es compatible con que $\mathrm{C}_{1}$ sea

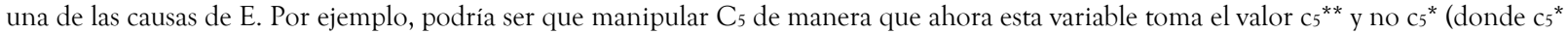
$\neq \mathrm{C}_{5}^{* *}$ mientras que el valor de $\mathrm{C}_{1}$ sigue siendo $\left.\mathrm{C}^{*}\right)$, E toma el valor $\mathrm{e}^{* *}$ que es distinto de $\mathrm{e}^{*}$. En otras palabras, $\mathrm{c}_{1}^{*}$ no siempre nos arrojaría $\mathrm{e}^{*}$ como resultado ya que el valor que $\mathrm{C}_{1}$ tome en cada caso dependerá de los valores que el resto de las variables causales tomen.
} 
Hablar de manipular variables en un grupo de objetos $\mathrm{u}$ organismos en el contexto de una caracterización de una noción causal podría llevarnos a pensar que esta noción causal tiene una aplicación limitada a aquellas situaciones en las cuales es factible o practicable manipular selectivamente una o más variables; es decir, situaciones de experimentación controlada. Pero, como Woodward mismo señala, esto sería una conclusión apresurada, puesto que su caracterización de causa se refiere al valor que tomaría un efecto E si se manipulasen una o más variables causales. La definición de causa de Woodward no requiere ni que tales manipulaciones de hecho se lleven a cabo, ni que sea factible llevarlas a cabo, ni mucho menos que puedan ser selectivas (por ejemplo, que se pueda cambiar sólo una variable a la vez). Claro, para poder estar en posición de afirmar razonablemente que una variable es causa de otra es necesario que existan buenas razones para pensar que, si alguna manipulación se llevara a cabo en la presunta variable-causa, algunos cambios en la presunta variableefecto se darían.

Hasta aquí sólo hemos bosquejado la noción de causa de Woodward; procedemos ahora a proponer una caracterización más precisa de la idea que una variable es causa específica de otra variable.

\subsection{Especificidad causal: una propuesta}

Sea $G$ un grupo de varios subgrupos, $G_{1}, \ldots, G_{p}$, de organismos de la misma especie, familia o género (por ejemplo, G podría ser un grupo de subgrupos de vertebrados, o bien el grupo de todas las especies de Drosophila, o el grupo de poblaciones de organismos de la misma especie, etc.). Adicionalmente, sea $\mathrm{C}$ una variable-causa de una variable-efecto $\mathrm{T}$ (donde 'causa' tiene el sentido woodwardiano y ' $\mathrm{T}$ ' refiere a un rasgo de los organismos de los subgrupos de $G$ ). $\left\{c_{1}, \ldots, c_{n}\right\}$ y $\left\{t_{1}, \ldots, t_{m}\right\}$ son todos los valores posibles que $\mathrm{C}$ y $\mathrm{T}$ pueden tener en $\mathrm{G}$. Para cada subgrupo $\mathrm{G}_{\mathrm{i}}$ de $\mathrm{G}(\mathrm{i}=1, \ldots, \mathrm{p})$, existen conjuntos de variables causales contextuales $\mathrm{B}_{\mathrm{i}}{ }^{1}, \ldots, \mathrm{B}_{\mathrm{i}}{ }^{\mathrm{q}}$, cada una de las cuales es una variable-causa de $\mathrm{T}$ distinta de $\mathrm{C}$ y tiene un rango de valores típicos de $\mathrm{G}_{\mathrm{i}}$ (en la producción de $\mathrm{T}$ ). Entonces diremos que $\mathrm{C}$ es una causa específica de T en G sólo si

(a) para toda $G_{i}$ de $G$ y para cada uno de los valores $c_{j}$ que $C$ puede tomar en $G(j=1, \ldots, n), c_{j}$, junto con los valores típicos de las variables contextuales $B_{i}{ }^{1}, \ldots, B_{i}{ }^{q}$, causan $t_{r}(r=1, \ldots, m)$ en $G_{i}$, (en el sentido woodwardiano de 'causa'), y

(b) no existe ningún otro valor $c_{k}(\mathrm{j} \neq \mathrm{k})$ de $\mathrm{C}$ tal que $\mathrm{c}_{\mathrm{k}}$, junto con los valores típicos de las variables contextuales $B_{i}{ }^{1}, \ldots, B_{i}{ }^{\mathrm{q}}$, causan $t_{r}$ en $G_{i \cdot}{ }^{7}$

La Figura 1 ilustra un caso en el que se puede decir que $\mathrm{C}$ es una causa específica de $\mathrm{T}$.

Figura 1

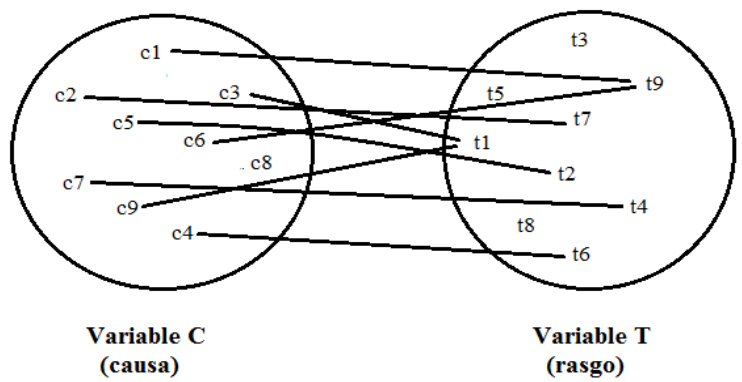

C es una causa específica de $T$.

\footnotetext{
${ }^{7}$ Esta noción de causa específica está inspirada en la que propone el mismo Woodward (2010).
} 
De acuerdo con esta noción de causa específica, por ejemplo, el gen Pax-6/eyeless, involucrado en el desarrollo temprano de los ojos tanto de vertebrados, como de cefalópodos y artrópodos es una causa (en el sentido woodwardiano) del desarrollo de cada uno de estos tipos de ojos, pero no es causalmente específico para ninguno de ellos; por ejemplo, si se elimina el gen Pax-6/eyeless de un cefalópodo (llamémoslo, GP6E $\mathrm{C}$, que es el valor que el gen Pax-6 eyeless toma en el grupo de los cefalópodos) y se inserta el gen Pax-6/eyeless de un artrópodo $\left(\mathrm{GP}_{\mathrm{E}} \mathrm{E}\right)$ en la secuencia apropiada del desarrollo del ojo de un cefalópodo (es decir, en el conjunto de variables causales contextuales típicas del desarrollo del ojo de un cefalópodo), el resultado final es un ojo de cefalópodo perfectamente bien formado, y no un ojo de artrópodo -por lo que el gen Pax-6/eyeless no es causalmente específico del ojo del cefalópodo. ${ }^{8}$

Usando esta noción de causa específica, caracterizamos ahora las nociones de paralelismo y convergencia, algunas de las cuales, como veremos más adelante, probablemente se adecúan a la manera en que un gran número de biólogos comparados las usan; por ejemplo, que dos rasgos son paralelos sólo si los grupos que presentan esos rasgos semejantes son filogenéticamente cercanos (Arendt \& Reznick 2007). ${ }^{9}$

\subsection{Paralelismo morfológico}

El rasgo $T_{1}$ de la especie $S_{1}$ es evolutivamente paralelo al rasgo $T_{2}$ de $S_{2}$ si y sólo si

(a) $T_{1}$ y $T_{2}$ son semejantes pero homoplásicos, $y$

(b) algunas porciones tardías del desarrollo de $T_{1}$ y de $T_{2}$ son homólogas y causalmente específicas para cada uno de esos rasgos.

La Figura 2 ilustra la manera en que se consideran los tiempos del desarrollo de un rasgo T.

Figura 2

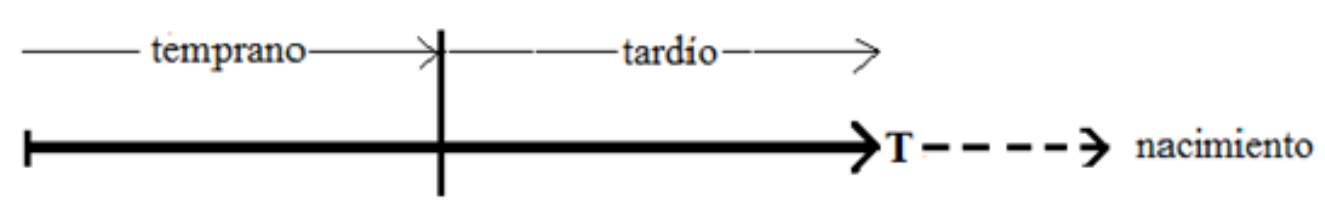

Tiempo del desarrollo del rasgo $\mathrm{T}$.

Cuándo empieza y cuándo termina el desarrollo de un rasgo pueden o no ser muy claramente definibles, pero en la mayoría de los casos se puede indicar aproximadamente cuándo se empiezan a desarrollar, por ejemplo, los ojos de un vertebrado, los dígitos, cada uno de los órganos internos, etc. ${ }^{10}$

\subsection{Convergencia}

El rasgo $T_{1}$ de la especie $S_{1}$ es convergente al rasgo $T_{2}$ de $S_{2}$ si y sólo si

(a) $\mathrm{T}_{1}$ y $\mathrm{T}_{2}$ son semejantes pero homoplásicos,

(b) ninguna porción tardía del desarrollo de $T_{1}$ es homóloga a ninguna porción tardía del desarrollo de $T_{2}, y$

(c) si alguna porción temprana del desarrollo de $T_{1}$ es homóloga a una porción temprana del desarrollo de $T_{2}$, entonces ninguna de esas porciones son causalmente específicas de $T_{1}$ o de $T_{2}$ (según sea el caso). ${ }^{11}$

\footnotetext{
${ }^{8}$ Lo mismo sucede en el caso del desarrollo de los ojos de los artrópodos y de los vertebrados.

${ }^{9}$ Véase nota 2.

${ }^{10}$ Nótese que, en el caso de algunos rasgos morfológicos al igual que para muchos sistemas cognitivos, sus desarrollos ontogenéticos continúan después del nacimiento y, en algunos casos, en la "adultez".
} 
Así, de acuerdo con mi propuesta, las principales diferencias entre paralelismo y convergencia son las siguientes:

A. En el caso del paralelismo, por lo menos una de las porciones tardías del desarrollo de $T_{1}$ en $S_{1}$ es homóloga a una correspondiente porción tardía del desarrollo de $\mathrm{T}_{2}$ en $\mathrm{S}_{2}$, las cuales son también causalmente específicas de $T_{1} \mathrm{o} T_{2}$, según sea el caso (algo que no sucede en el caso de la convergencia); lo que indica que por lo menos algunas de las razones principales por las cuales $T_{1}$ y $T_{2}$ son semejantes refieren a estos factores ontogenéticos de herencia $y / 0$ continuidad.

B. En el caso de la convergencia, ninguna homología ontogenética (ya sea a nivel de los genes o de las redes genéticas regulatorias) explicaría las semejanzas entre $T_{1}$ y $T_{2}$, ya que tales homologías, o bien no existen, o si existen, no son causalmente específicas ni de $T_{1}$ ni de $T_{2}$. Así que la explicación más probable de la existencia de las semejanzas entre $T_{1}$ y $T_{2}$ se encontraría en la presencia de semejanzas en los factores ambientales responsables de la fijación de $T_{1}$ en una población ancestral de la especie $S_{1}$ y de $T_{2}$ en otra población ancestral de $S_{2}$, es decir, la selección natural. Si esto es así, entonces decir que ciertos rasgos de dos grupos de organismos son convergentes involucra referirse a las historias evolutivo-adaptativas (no necesariamente muy antiguas) de $S_{1}$ y $S_{2}$, afirmando que existieron presiones ambientales semejantes en respuesta a las cuales se fijaron fenotipos semejantes, y que estas semejanzas no tienen una explicación en términos de la existencia de homologías ontogenéticas.

Pasamos ahora a considerar la noción de homología profunda y a explicar cómo se diferencia del paralelismo y de la convergencia.

\subsection{Homología profunda morfológica}

El rasgo $T_{1}$ de la especie $S_{1}$ es profundamente homólogo al rasgo $T_{2}$ de $S_{2}$ si y sólo si una porción temprana del desarrollo de $T_{1}$ es homóloga a una porción temprana del desarrollo de $T_{2}$.

La distinción entre la homología profunda y el paralelismo -tal y como las hemos caracterizado- no tiene que ser tajante; puede haber casos límite, dependiendo de qué tan finamente queramos trazar la línea divisoria entre factores causales tempranos y tardíos. Y aunque la distinción entre paralelismo y homología profunda admite de grados - una consecuencia que la mayoría de los biólogos y filósofos de la biología probablemente encontrarían aceptable (Hall 2007, Leander 2008, Wake et al. 2011, Powell 2012, Ochoa \& Rasskin-Gutman 2015)- la decisión con respecto al lugar exacto en el cual trazar la línea divisoria entre factores tempranos y tardíos (cuando tal decisión es necesaria) dependerá casi siempre de factores pragmáticos; lo cual no es un problema serio. Muchas de las decisiones en la biología y en las ciencias naturales son de este tipo, en el sentido que ningún "hecho duro" las determina, y se usan procedimientos heurísticos distintos dependiendo del contexto disciplinar de toma de decisión.

Por otra parte, la principal diferencia entre la homología profunda, por una parte, y el paralelismo y la convergencia, por otra, es la siguiente: si $T_{1}$ y $T_{2}$ son profundamente homólogas, entonces una de las porciones tempranos del desarrollo de $\mathrm{T}_{1}$ tiene que ser homóloga a una de las porciones tempranas del desarrollo de $\mathrm{T}_{2}$ (mientras que esto no es necesario, aunque sí posible, en el caso del paralelismo y

\footnotetext{
${ }^{11}$ Como veremos más adelante, añado esta tercera cláusula a fin de poder distinguir la convergencia de la homología profunda.
} 
de la convergencia). ${ }^{12}$ La Tabla 1 resume las semejanzas y diferencias entre paralelismo, convergencia y homología profunda, según mi propuesta:

Tabla 1

\begin{tabular}{|c|c|c|c|c|c|c|c|}
\hline \multirow{3}{*}{$\begin{array}{l}\text { Rasgos } \mathrm{T} 1 \text { de } \\
\text { especie S1, y } \\
\text { T2 de especie S2 }\end{array}$} & \multirow{3}{*}{$\begin{array}{l}\text { Seme- } \\
\text { janza }\end{array}$} & \multirow{3}{*}{$\begin{array}{c}\text { Sinapo- } \\
\text { morfia }\end{array}$} & \multirow{3}{*}{$\begin{array}{l}\text { Selecc. } \\
\text { Natural }\end{array}$} & \multirow{3}{*}{$\begin{array}{c}\text { Cercania } \\
\text { Filo- } \\
\text { genética }\end{array}$} & \multicolumn{3}{|c|}{$\begin{array}{c}\text { CAUSAS DEL } \\
\text { DESARROLLO DE } \\
\text { T1 Y T2 }\end{array}$} \\
\hline & & & & & $\begin{array}{l}\text { TEM- } \\
\text { PRANAS }\end{array}$ & \multicolumn{2}{|c|}{ TARDIAS } \\
\hline & & & & & $\begin{array}{c}\text { Homo- } \\
\text { logas }\end{array}$ & $\begin{array}{l}\text { Homo } \\
\text {-logas }\end{array}$ & $\begin{array}{l}\text { Espe- } \\
\text { cifica }\end{array}$ \\
\hline PARALELISMO & sí & NO & No & $\begin{array}{l}\text { Típica- } \\
\text { mente } \\
\text { Sí }\end{array}$ & $\begin{array}{l}\text { No } \\
\text { Nec }\end{array}$ & SI & SI \\
\hline CONVERGENCIA & si & No & sí & No Nec & $\begin{array}{l}\text { No } \\
\text { Nec }\end{array}$ & No & $\begin{array}{l}\text { No } \\
\text { Nec }\end{array}$ \\
\hline $\begin{array}{l}\text { HOMOLOGIA } \\
\text { PROFUNDA }\end{array}$ & No $\mathrm{Nec}$ & No $\mathrm{Nec}$ & No $\mathrm{Nec}$ & $\begin{array}{l}\text { Tipica- } \\
\text { mente } \\
\text { NO }\end{array}$ & SI & $\begin{array}{l}\text { No } \\
\text { Nec }\end{array}$ & $\begin{array}{l}\text { No } \\
\text { Nec }\end{array}$ \\
\hline
\end{tabular}

Semejanzas y diferencias entre el paralelismo, la convergencia y la homología profunda.

\section{Una motivación de la propuesta}

En este punto surge una pregunta crucial acerca de mi propuesta: ¿por qué deberíamos distinguir el paralelismo de la homología profunda en términos de una distinción entre las porciones tardías y las tempranas de los desarrollos correspondientes? La respuesta a esta pregunta está relacionada con una discusión actual en la biología evolutiva del desarrollo sobre si existen o no ciertas tendencias macro evolutivas en grandes grupos de animales; tendencias derivadas de la estructura causal del desarrollo ontogenético. ${ }^{13}$ Hay por lo menos tres posiciones distintas a este respecto:

4.1. La primera tesis, a veces llamada "el modelo de la conservación temprana" (Piasecka et al. 2013), o "el modelo del embudo" está relacionada con lo que, según Wimsatt, es una formulación de la ley de von Bauer; a saber, que

[c]omo resultado deberíamos esperar que la evolución sea crecientemente conservadora en las etapas más tempranas del desarrollo de modo que los rasgos que se expresan más tempranamente en el desarrollo sean probablemente más antiguos [...] que los rasgos que se expresan más tardíamente en el desarrollo (Wimsatt 1986, p. 198; el énfasis es mío).

La idea es que, entre más temprano en el desarrollo ocurra una mutación, más probable es que ésta tenga consecuencias más dañinas y extensas (o incluso letales) -que una mutación que ocurra en una etapa más tardía; lo cual, según Wimsatt, tiene consecuencias evolutivas a largo plazo, por ejemplo, que existan sólo unas cuantas variaciones en los rasgos que aparecen en las etapas más tempranas del

\footnotetext{
${ }^{12}$ Queda pendiente una pregunta crucial, a saber, ¿cómo se puede determinar cuándo porciones de dos redes genéticas regulatorias son homólogas? Aunque aquí no puedo entrar en esta controversia, sin embargo puedo sí señalar en la dirección del trabajo seminal de Ehab Abouheif (1999), y de las investigaciones más recientes de autores como Erwin y Davidson (2007).

${ }^{13}$ Powell (2012) también argumenta que la distinción paralelismo/convergencia es importante ya que tiene una relevancia directa respecto de la discusión sobre cuánta contingencia existe en la macroevolución orgánica.
} 
desarrollo de ciertos grandes grupos de organismos- esto es, en los rasgos constitutivos del llamado 'plan corporal' (Bauplan) característico de estos grupos (Davidson \& Erwin 2006).

Si esto fuera correcto, entonces la distinción aquí propuesta entre aquellos rasgos que son profundamente homólogos y los que son paralelos, tendría una conexión probabilística directa con la distinción entre (respectivamente) aquellos rasgos algunas de cuyas semejanzas descansan parcialmente en homologías del desarrollo que son evolutivamente antiguas, y aquellos rasgos cuyas semejanzas se deben a homologías del desarrollo que son evolutivamente más recientes.

William Wimsatt (1986), por ejemplo, nos dice que "el desarrollo apropiado de rasgos en etapas posteriores presupone o depende de la presencia de ciertos rasgos en etapas más tempranas, y que si esos rasgos no están presentes en etapas tempranas, esto tiene un alta probabilidad de causar una disfunción en etapa o etapas tardías" (1986, p. 195, énfasis mío). ${ }^{14} \mathrm{Si}$ esto fuera correcto, por ejemplo, para el desarrollo de la mayoría de los vertebrados (o los artrópodos o los cefalópodos), entonces, afirma Wimsatt, a largo plazo surgiría una tendencia macro evolutiva según la cual los rasgos evolutivamente más conservados en la mayoría de los linajes de metazoarios tenderían a ser aquéllos que aparecen más tempranamente en el desarrollo ontogenético, mientras que los rasgos que aparecen más tardíamente tenderían a ser más evolucionables, en el sentido de presentar un potencial para un número mayor de variaciones viables en estos linajes.

4.2. Por otra parte, existe una segunda tesis acerca de la existencia de tendencias macro evolutivas derivadas de (o conectadas con) la(s) estructura(s) causal(es) del desarrollo. Esta tesis descansa en un modelo del desarrollo conocido como "reloj de arena". El nombre de esta tesis deriva de la forma del dibujo que mejor representa visualmente la idea central (Figura 3).

Figura 3

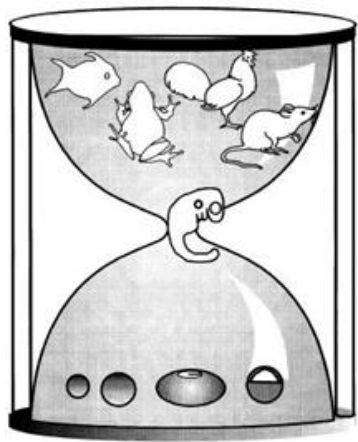

El modelo de desarrollo como un reloj de arena (Richarson et al. 1997).

La idea de este modelo es que existen tres etapas del desarrollo en por lo menos algunos de los grandes grupos de metazoarios (por ejemplo, los vertebrados). Una de estas etapas, la intermedia, se caracteriza por presentar muy poca variación entre los embriones de las diferentes especies comprendidas dentro de cada uno de esos grupos. Esta etapa se conoce como la etapa filotípica, mientras que en las otras dos etapas existe una mayor variación embrionaria interespecífica. En los vertebrados, esta etapa intermedia también se conoce como la faríngula, pero hay cierta evidencia para pensar que también existe una etapa embrionaria de escasa variación en otros organismos, por ejemplo, en especies de

\footnotetext{
${ }^{14}$ Para ser más precisos, Wimsatt (1986) piensa que (con ciertas excepciones que se generan por la existencia de la canalización y otros mecanismos ontogenéticos) existe una relación de dependencia probabilística exponencial entre una etapa tardía y una más temprana en el desarrollo de la mayoría de los metazoarios.
} 
Drosophila. Algunos biólogos (por ejemplo, Sander 1983 y Raff 1996) han argumentado que, durante esta etapa, hay ciertos constreñimientos del desarrollo de los vertebrados que impiden la existencia de una mayor variación. Por ejemplo, durante la etapa de la faríngula, la mayoría de los módulos ontogenéticos dependen marcadamente para su correcta operación de la activación de otros módulos, de manera tal que si el corazón no se desarrolla en un lugar determinado en el embrión, esto puede afectar el desarrollo de los ojos, y hay muchos otros ejemplos como éste. ${ }^{15}$

En términos evolutivos, esto significaría que los rasgos que se desarrollan durante la etapa filotípica en los organismos relevantes (por ejemplo, los vertebrados) tenderán a presentar pocas variaciones interespecíficas en las poblaciones de organismos adultos, mientras que aquéllos que se desarrollan en las etapas anteriores o posteriores probablemente presentarán muchas más variaciones. Esto también sugiere que es probable que, por ejemplo, en los vertebrados, existan por lo menos dos tipos de tendencias macroevolutivas correspondientes a los grados de evolucionabilidad que presentan los rasgos que se desarrollan durante la etapa filotípica, en contradistinción con los que se desarrollan en la etapa post filotípica. Así, según este modelo, a largo plazo, tenderá a existir un grado amplio de conservación evolutiva en los rasgos que se desarrollan en la primera etapa, y un grado mucho mayor de variación en los rasgos de la segunda etapa.

4.3. Finalmente, está la idea de que probablemente no existe ninguna tendencia macro evolutiva derivada de la estructura causal del desarrollo ontogenético en grandes grupos de animales. Jeff Arendt y David Reznick (2007) parecen inclinarse por esta tesis al cuestionar la idea que existe una correlación probabilística entre compartir mecanismos del desarrollo y una relación filogenética cercana, al igual que entre rasgos semejantes cuyos mecanismos del desarrollo son distintos y una relación lejana, afirmando que:

Organismos que están cercanamente relacionados con frecuencia evolucionan el mismo fenotipo a través de diferentes mecanismos [del desarrollo] y organismos lejanamente relacionados con frecuencia evolucionan el mismo fenotipo a través del mismo mecanismo (Arendt \& Reznick 2007, p. 27).

Su argumento, sin embargo, no muestra que la probabilidad sea alta que los organismos de especies (taxones, linajes) cercanamente relacionadas desarrollen rasgos muy semejantes por medio de diferentes genes o diferentes redes genéticas regulatorias; ni tampoco muestra que sea alta la probabilidad de que especies lejanamente relacionadas desarrollen rasgos semejantes por medio de genes y/o redes genéticas homólogos. Lo único que muestran ellos es que existen algunos casos que tienen estas características, a partir de los cuales brincan a la conclusión que esto es muy frecuente en la naturaleza; una conclusión que está débilmente respaldada por la evidencia que ellos proporcionan. Incluso ellos mismos conceden con renuencia que:

La asociación entre afinidad taxonómica y la semejanza del mecanismo que causa la evolución independiente de la semejanza fenotípica podría ser probabilística -podría ser más probable que especies más cercanamente relacionadas evolucionaran una semejanza fenotípica a través de un mismo mecanismo [del desarrollo] que especies más lejanamente relacionadas-. (Arendt \& Reznick 2007, p. 31; el énfasis es mío)

Así, lo único claro hasta el momento es que aún no hay un consenso entre los biólogos evolutivos del desarrollo con respecto a si existe o no alguna tendencia macro evolutiva de algún tipo derivada de la

\footnotetext{
${ }^{15}$ Otro ejemplo: una falla en el desarrollo del mesodermo en una determinada parte del embrión conduce a malformaciones de los riñones, las extremidades y la cola. Galis y Metz (2001) han mostrado que la faríngula es uno de los estadios del desarrollo más vulnerables, estudiando los patrones de malformaciones múltiples en diferentes órganos en humanos. Existe también evidencia molecular que los patrones de expresión genética dentro de un cierto grupo tienen la forma de un reloj de arena y que las etapas en las cuales hay una mayor semejanza interespecífica son la faríngula en los vertebrados y el período de segmentación en especies de Drosophila. (Domazet-Lošo \& Tautz 2010, Kalinka et al. 2010, Irie \& Kuratani 2011, Wang et al. 2013).
} 
estructura causal del desarrollo y, si existe, de qué tipo sería (tipo reloj de arena o tipo embudo, o bien de algún otro tipo aún no conceptualizado). Lo que sí se puede afirmar aquí es que si existe alguna de las tendencias macroevolutivas examinadas aquí, entonces es probable que exista una conexión entre paralelismo/homología ontogenética de reciente cuño, y homología profunda/homología ontogenética antigua -tal y como he caracterizado las nociones de paralelismo y homología profunda-.

Un último punto importante: tal y como entiendo la convergencia, y hasta dónde puedo visualizarlo, no es ni necesario ni probable que exista una conexión probabilística entre la convergencia y la existencia de una homología ontogenética antigua. Todo lo que se desprende de la caracterización de convergencia es que, si dos rasgos son convergentes entonces cada uno de ellos se fijó en las poblaciones ancestrales relevantes por la acción de presiones ambientales semejantes. Pero nada en la definición nos dice que tales rasgos se fijaron, en términos evolutivos, hace poco o hace mucho. Es en este punto en el que mi manera de entender la convergencia difiere de la forma en que normalmente usan esta noción muchos biólogos evolutivos. Por otra parte, la conexión entre convergencia y fijación por presiones ambientales semejantes es un punto de acuerdo entre la noción aquí propuesta y el uso común de tal noción.

\section{Bibliografía}

Abouheif, E. (1999), "Establishing Homology Criteria for Regulatory Gene Networks: Prospects and Challenges", en Bock, G. R. y G. Cardew (eds.), Homology, Chichester: Wiley (Novartis Foundation Symposium 222), pp. 207-225.

Arendt, J. y D. Reznick (2007), "Convergence and Parallelism Reconsidered: What Have We Learned About the Genetics of Adaptation?", Trends in Ecology and Evolution 23: 26-32.

Davidson, E.H. y D.H. Erwin (2006), "Gene Regulatory Networks and the Evolution of Animal Body Plans", Science 311: 796-800.

de Pinna, M.G.G. (1991), “Concepts and Tests of Homology in the Cladistic Paradigm”, Cladistics 7: 367-394.

Domazet-Lošo, T. y D. Tautz (2010), "A Phylogenetically Based Transcriptome Age Index Mirrors Ontogenetic Divergence Patterns”, Nature 468: 815-818.

Duboule, D. (1994), "Temporal Colinearity and the Phylotypic Progression: A Basis for the Stability of a Vertebrate Bauplan and the Evolution of Morphologies through Heterochrony”, Development (Suppl.): 135-142.

Erwin, D.H. y E.H. Davidson (2009), "The Evolution of Hierarchical Gene Regulatory Networks", Nature Reviews Genetics 10: 141-148.

Galis, F. y J.A. Metz (2001), "Testing the Vulnerability of the Phylotypic Stage: On Modularity and Evolutionary Conservation”, Journal of Experimental Zoology/Molecular Developmental Evolution 291: 195-204.

García C.L. (en prensa), “"El paralelismo y la especificidad causal en el desarrollo tardío: examen de la propuesta de Russell Powell con respecto al paralelismo evolutivo", Teorema 37(1).

García C.L. (2010), "Functional Homology and Functional Variation in Evolutionary Cognitive Science", Biological Theory 5: 124-135.

Hall, B.K. (2003), "Descent with Modification: The Unity Underlying Homology and Homoplasy as Seen Through an Analysis of Development and Evolution”, Biological Review 78: 409-433.

Hall, B.K. (2007), "Homoplasy and Homology: Dichotomy or Continuum?", Journal of Human Evolution 52: 473-479.

Irie, N. y S. Kuratani (2011), "Comparative Transcriptome Analysis Reveals Vertebrate Phylotypic Period During 
68 |Claudia Lorena García

Organogenesis”, Nature Communications 2: 248.

Kalinka, A. T., Varga, K.M., Gerrard, D.T., Preibisch, S., Corcoran, D.L., Jarrells, J., Ohler,U., Bergman, C.M. y P. Tomancak (2010), "Gene Expression Divergence Recapitulates the Developmental Hourglass Model”, Nature 468: 811-814.

Laubichler, M.D. (2000), "Homology in Development and the Development of the Homology Concept", American Zoologist 40: 777-788.

Leander, B.S. (2008), "Different Modes of Convergent Evolution Reflect Phylogenetic Distances: A Reply to Arendt and Reznick", Trends in Ecology and Evolution 23: 481-482.

Müller, G.B. (2003), “Homology: The Evolution of Morphological Organization”, en Müller, G. y S.A. Newman (eds.), The Origination of Organismal Form: Beyond the Gene in Developmental and Evolutionary Biology, Cambridge, Massachusetts: MIT Press, pp. 51-69.

Ochoa, C. y D. Rasskin-Gutman (2015), “Evo-devo Mechanisms Underlying the Continuum between Homology and Homoplasy”, Journal of Experimental Zoology/ Molecular Developmental Evolution 324B: 91-103.

Piasecka, B., Lichocki, P., Moretti, S., Bergmann, S. y M. Robinson-Rechavi (2013), "The Hourglass and the Early Conservation Models-Co-Existing Patterns of Developmental Constraints in Vertebrates”, PLoS Genetics 9(4): e1003476.

Powell, R. (2012), "Convergent Evolution and the Limits of Natural Selection”, European Journal for the Philosophy of Science 2: 355-373.

Raff, R.A. (1996), The Shape of Life: Genes, Development and the Evolution of Animal Form, Chicago: University of Chicago Press.

Sander, K. (1983), "The Evolution of Patterning Mechanisms: Gleaning from Insect Embryogenesis and Spermatogenesis" en Goodwin, B.C., Holder, N. y C.C. Wylie (eds.), Development and Evolution, Cambridge: Cambridge University Press, pp. 137-159.

Scotland, R. (2011), "What is Parallelism?", Evolution and Development 13: 214-227.

Shubin, N., Cliff, T. y S. Carroll (2009), "Deep Homology and the Origins of Evolutionary Novelty”, Nature 457: 818823.

Simpson, G.G. (1961), Principles of Animal Taxonomy, New York: Columbia University Press.

Sommer, R.J. (2008), “Homology and the Hierarchy of Biological Systems”, BioEssays 30: 653-658.

Striedter, G.F. y R.G. Northcutt (1991), "Biological Hierarchies and the Concept of Homology”, Brain, Behavior and Evolution 38: 177-189.

Wagner, G.P. (1989), "The Bological Homology Concept”, Annual Review of Ecology and Systematics 20: 51-69.

Wake, D.B., Wake, M.H. y C.D. Specht (2011), "Homoplasy: From Detecting Pattern to Determining Process and Mechanism of Evolution”, Science 331: 1032-1035.

Wang, Z., Pascual-Anaya, J., Zadissa, A., Li, W., Niimura, Y., Huang, Z., Li, C., White, S., Xiong, Z., Fang, D., Wang, B., Ming, Y., Chen, Y., Zheng, Y., Kuraku, S., Pignatelli, M., Herrero, J., Beal, K., Nozawa, M., Li, Q., Wang, J., Zhang, H., Yu, L., Shigenob, S., Wang, J., Liu, J., Flicek, P., Searle, S., Wang, J., Kuratani, S., Yin, Y., Aken, B., Zhang, G. y N. Irie (2013), "The Draft Genomes of Soft-Shell Turtle and Green Sea Turtle Yield Insights into the Development and Evolution of the Turtle-Specific Body Plan”, Nature Genetics 45: 701-706.

Waters, C.K. (2007), “Causes that Make a Difference”, Journal of Philosophy 104: 551-579.

Wimsatt, W.C. (1986), "Developmental Constraints, Generative Entrenchment and the Innate-Acquired Distinction”, en Bechtel, W. (ed.), Integrating Scientific Disciplines, Dordrecht: Martinus Nijhoff, pp. 185-208. 
Woodward, J. (2003), Making Things Happen: A Theory of Causal Explanation, Oxford: Oxford University Press.

Woodward, J. (2010), “Causation in Biology: Stability, Specificity, and the Choice of Levels of Explanation”, Biology $\mathcal{E}$ Philosophy 25: 287-318. 